\title{
Inhibition of LncRNA MALAT1 Attenuates Cerebral Ischemic Reperfusion Injury via Regulating AQP4 Expression
}

\author{
Jing Jin ${ }^{a}$ Hongwei Wang ${ }^{b}$ Xiaoxiao Zheng ${ }^{c, d}$ Shangzhi Xie ${ }^{c, d}$ Li Zheng $^{c, d}$ \\ Renya Zhan ${ }^{\text {a }}$ \\ aDepartment of Neurosurgery, The First Affiliated Hospital, Zhejiang University, School of Medicine, \\ Hangzhou, China; 'bepartment of Anesthesiology, Tongde Hospital of Zhejiang Province, Hangzhou, China; \\ 'Cancer Institute of Integrated Traditional Chinese and Western Medicine, Zhejiang Academy of Traditional \\ Chinese Medicine, Tongde Hospital of Zhejiang Province, Hangzhou, China; dDepartment of Medical Oncology, \\ Tongde Hospital of Zhejiang Province, Hangzhou, China
}

\section{Keywords}

Cerebral ischemia reperfusion injury - MALAT1 .

Aquaporin-4

\begin{abstract}
Stroke is one of the leading causes of mortality and disability worldwide. Long noncoding RNAs (IncRNAs) including MALAT1 have been shown to have critical roles in cerebral ischemia reperfusion injury (CIRI). However, the underlying mechanism of MALAT1 in CIRI has not been elucidated. The present study aimed to investigate the function and potential regulatory mechanism of MALAT1 in cerebral ischemic reperfusion injury. We established the middle cerebral artery occlusion (MCAO) model and oxygen-glucose deprivation/ reoxygenation $(\mathrm{OGD} / \mathrm{RX})$ model in vivo and in vitro, and then Cell Counting Kit-8 (CCK-8), RT-qPCR, flow cytometry analysis, lactate dehydrogenase (LDH) analysis, and 2,3,5-triphenyltetrazolium chloride (TTC) staining were used to examine cell viability, MALAT1, aquaporin-4 (AQP4) expression, LDH release, and infarct volume, respectively. The level of AQP4 was remarkably upregulated in CIRI $24 \mathrm{~h} / 48 \mathrm{~h}$ or OGD/RX 24 $\mathrm{h} / 48 \mathrm{~h}$ compared with the sham group. Knockdown of AQP4
\end{abstract}

karger@karger.com www.karger.com/ene

Karger $\stackrel{\text { ' }}{5}$

GOPEN ACCESS
(C) 2020 The Author(s)

Published by S. Karger AG, Basel

This is an Open Access article licensed under the Creative Commons Attribution-NonCommercial-4.0 International License (CC BY-NC) (http://www.karger.com/Services/OpenAccessLicense), applicable to the online version of the article only. Usage and distribution for commercial purposes requires written permission. could alleviate OGD/RX-induced injury through enhancing cell viability and reducing $L D H$ release and the rate of apoptotic cells. Furthermore, we found that MALAT1 was also increased in OGD/RX $24 \mathrm{~h} / 48 \mathrm{~h}$ and silencing of MALAT1 could decrease AQP4. Inhibition of MALAT1 could also protect OGD/RX-induced injury, while the protective effect of MALAT1 siRNA on cerebral ischemic reperfusion was disappeared after transfection with AQP4 plasmid, indicating that MALAT1 may play a protective role in brain stroke through regulating AQP4. Taken together, our study provides evidence that MALAT1 is involved in ischemic stroke by inhibiting AQP4. Therefore, MALAT1 may serve as a potential target for therapeutic intervention in ischemic brain injury.

C 2020 The Author(s)
Published by S. Karger AG, Basel

\section{Introduction}

Stroke is a leading cause of long-term disability and high mortality worldwide, and approximately $80-85 \%$ of strokes belong to ischemic stroke, which usually ascribes to embolic or thrombotic occlusion of the cerebral artery $[1,2]$. Recently, thrombolytic therapy is the most effec- 
tive clinical treatment to restore blood supply for ischemic stroke as early as possible, but cerebral ischemiainduced brain cell damage referred to as ischemia/reperfusion (I/R) injury may aggravate reperfusion following thrombolysis treatment $[3,4]$. Therefore, a better understanding of the mechanisms involved in cerebral I/R injury may provide crucial clues for ischemic stroke treatment.

Long noncoding RNAs (lncRNAs), a set of noncoding RNAs of length $>200$ nucleotides (nt), have regulatory roles in diverse cellular contexts and many biological processes such as cell proliferation, apoptosis, differentiation, autophagy, angiogenesis, and immune responses [5, $6]$. It has been reported that $\operatorname{lncRNAs}$ were involved in ischemic stroke [6]. For instance, downregulation of lncRNA ROR could alleviate cerebral hypoxia/reoxygenation-induced injury in PC12 cells [7]. Interference of lncRNA H19 could significantly prevent neurogenesis in ischemic stroke by decreasing the volume of brain tissue loss and the fluorescence intensity of neurogenesis-related proteins [8]. More interestingly, MALAT1 was significantly upregulated in both oxygen-glucose deprivation (OGD) endothelial cells and middle cerebral artery occlusion (MCAO) mouse models of stroke [9]. Knockdown of MALAT1 results in larger brain infarct size, worse neurological scores, and reduced sensorimotor functions post-MCAO [10]. Since cerebral vasculature is important in improving clinical outcomes in the postischemic recovery phase, increasing MALAT1 in ischemic stroke suggested a protective and healing property of the ischemic brain.

Aquaporin-4 (AQP4), a family member of waterchannel proteins, could facilitate the transmembrane diffusion of water and is involved in cerebral ischemia reperfusion, cerebral edema, and water homeostasis. It is abundant and widely distributed in the brain and expressed in astrocytic extensions close to capillaries. It helps water pass through the blood-brain barrier (BBB). Furthermore, inhibition of AQP4 significantly reduces OGD/reoxygenation (RX)-induced astrocyte cell injury in vitro $[11,12]$ and decreases the volume of infarcted area associated with ischemic injury in vivo [13]. Therefore, modulating AQP4 expression in the brain may support a new therapeutic strategy for reducing cerebral I/R.

In this study, we investigated the protective effect of MALAT1 in the MCAO model in vivo and OGD/reperfusion-treated astrocytes in vitro, which is involved in the underlying mechanism mediated by the expression of AQP4. This study provided a new insight into the protective role of MALAT1 in cerebral ischemic reperfusion.

\section{Materials and Methods}

\section{Animals}

Six-week-old male C57BL/6J mice (20-25 g) were purchased from the Experimental Animal Center of Zhejiang University School of Medicine. All mice were housed in an environmentally controlled room under a 12-h light/dark cycle with ad libitum access to food and water. All animal experiments were approved by the First Affiliated Zhejiang Hospital, Zhejiang University of Medical Ethics Committee, and the Medical Faculty Ethics Committee of the First Affiliated Zhejiang Hospital, Zhejiang University, in accordance with the National Institutes of Health Guide for Care and Use of Laboratory Animals (NIH Publications, No. 8023, revised 1978).

\section{Primary Astrocyte Culture}

Primary astrocytes were prepared from a postnatal day 7 cerebellum in C57BL/6J mice, and the protocol used was as described previously [14]. The cells were plated at a density of $1 \times 106$ cells/ $\mathrm{mL}$ onto 96-well plates and cultured in poly-L-lysine-coated 35mm dishes with Dulbecco's modified Eagle's medium (DMEM) containing $10 \% \mathrm{FBS}$ at $37^{\circ} \mathrm{C}$ in $5 \% \mathrm{CO}^{2}$ in a humidified incubator.

\section{OGD/RX Model Establishment}

The primary astrocyte OGD model was established in accordance with a previous study [15]. In brief, the cells were transferred to glucose-free DMEM and cultivated in a humidified incubator with $95 \% \mathrm{~N}_{2}$ and $5 \% \mathrm{O}_{2}$ at $37^{\circ} \mathrm{C}$ for $6 \mathrm{~h}$. For reperfusion, the medium was replaced with high-glucose DMEM and incubated in a normoxic incubator for an additional $24 \mathrm{~h}$.

\section{Focal Cerebral Ischemia Reperfusion Injury Model}

We used MCAO to establish the focal cerebral ischemia reperfusion injury (CIRI) model; the procedure was described in our previous study [16]. A total of $24 \mathrm{C} 57 \mathrm{BL} / 6 \mathrm{~J}$ mice were randomly grouped as follows $(n=6)$ : blank group, sham group, CIRI $24 \mathrm{~h}$, CIRI $48 \mathrm{~h}$, and shMALAT1 (short hairpin RNA targeting MALAT1) + CIRI $48 \mathrm{~h}$. In brief, the mice were anesthetized with $4 \%$ chloral hydrate $(40 \mathrm{mg} / \mathrm{kg}$ ) (Sigma, St. Louis, MO, USA) and a 6-0 silicone-coated nylon monofilament (Doccol Corp., Redlands, CA, USA) was inserted into the left common carotid artery to occlude the MCA origin. After $1 \mathrm{~h}$, the suture was removed. The sham group animals received the same surgical exposure of the carotid arteries without MCAO. The mice were then anesthetized and decapitated to obtain the brain.

\section{Cell Counting Kit-8 Assay}

Cell viability was determined using the Cell Counting Kit- 8 (CCK-8) assays. In brief, the astrocyte cells were seeded onto 96well plates at a density of $3 \times 10^{3}$ cells/well. After the indicated treatment, about $10 \mu \mathrm{L}$ of CCK- 8 solution was added to each well and then incubated for 2-3 h. Cell viability was measured using an MRX II microplate reader (Dynex Technologies, Chantilly, USA). Relative cell viability was calculated as a percentage of untreated controls.

\section{Cell Transfection}

Astrocyte cells were transfected with lncRNA-MALAT1 siRNA, AQP4 siRNA, AQP4 plasmid, or the relative negative control siRNA (siRNA-NC) using the Lipofectamine 2000 (Invitrogen, 
Carlsbad, CA, USA) according to the manufacturer's protocol. After $6 \mathrm{~h}$ of transfection, MA-C cells underwent OGD/RX treatment ( $6 \mathrm{~h}$ OGD and $24 \mathrm{~h} \mathrm{RX).} \mathrm{The} \mathrm{primer} \mathrm{sequences} \mathrm{were} \mathrm{as} \mathrm{follows:}$

- MALAT1 siRNA:

- MALAT1-1-Homo-209:

- Forward: 5'-GGUGGUGGUAUUUAGAUAATT-3'

- Reverse: $5^{\prime}$-UUAUCUAAAUACCACCACCTT-3'

- MALAT1-2-Homo-1790:

- Forward: 5'-GCGUCAUUUAAAGCCUAGUTT-3'

- Reverse: 5'-ACUAGGCUUUAAAUGACGCTT-3'

- MALAT1-3-Homo-4443:

- Forward: 5'-GGGCUGACAUUAACUACAATT-3'

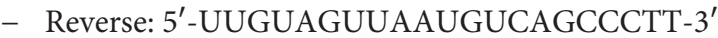

\section{Lactate Dehydrogenase Analysis}

Cytotoxicity Lactate Dehydrogenase (LDH) Assay Kit-WST can be used to measure the released LDH according to the manufacturer's protocol.

\section{Western Blot Assay}

Forty micrograms of protein per lane were separated via SDSPAGE, transferred onto polyvinylidene difluoride (PVDF) membranes (EMD Millipore), and blocked in 5\% skim milk at room temperature for $2 \mathrm{~h}$. Then, the membranes were incubated with primary antibodies against AQP4 (1:1,000; Abcam, Cambridge, MA, USA) overnight at $4^{\circ} \mathrm{C}$, followed by incubation with the horseradish peroxidase-labeled secondary antibody $(1: 2,000)$ at room temperature for $2 \mathrm{~h}$. Finally, protein blots were visualized using chemiluminescent ECL reagent (GE Healthcare, Piscataway, NJ, USA) and quantified by densitometry (Quantity One 4.5.0 software; Bio-Rad Laboratories, Inc.).

\section{Quantitative Reverse Transcription PCR}

Total RNA was extracted from cells using TRIzol ${ }^{\circledR}$ reagent (Invitrogen; Thermo Fisher Scientific, Inc.) according to the manufacturer's protocol. A TaqMan MicroRNA Reverse Transcription Kit (Applied Biosystems; Thermo Fisher Scientific, Inc.) was used for cDNA generation. Reaction conditions for RT were as follows: $50^{\circ} \mathrm{C}$ for $5 \mathrm{~min}$ and $80^{\circ} \mathrm{C}$ for $2 \mathrm{~min}$. An SYBR Premix Ex $\mathrm{Taq}^{\mathrm{TM}}$ II (TliRNaseH Plus) kit (Takara Bio, Inc.) was used to analyze the synthesized cDNA. U6 and GAPDH were used as internal controls for miRNA and mRNA expression, respectively. The thermocycling conditions were as follows: $95^{\circ} \mathrm{C}$ for $5 \mathrm{~min}$, followed by 38 cycles of denaturation at $95^{\circ} \mathrm{C}$ for $15 \mathrm{~s}$ and annealing/ elongation at $60^{\circ} \mathrm{C}$ for $30 \mathrm{~s}$. The relative gene expression was calculated using the $2^{-\Delta \Delta \mathrm{Cq}}$ method [17]. Primer sequences for PCR were as follows:

- MALAT1:

- Forward: 5' -TGTGACGCGACTGGAGTATG-3'

- Reverse: 5'-CAAAGGGACTCGGCTCCAAT-3'

- AQP4:

- Forward: 5'-GACAGACCCACAGCAAGG-3'

- Reverse: $5^{\prime}$-GCAAAGGGAGATGAGAACC-3'

- U6:

- Forward: 5'-CTCGCTTCGGCAGCACA-3'

- Reverse: $5^{\prime}$-AACGCTTCACGAATTTGCGT-3'

- $\beta$-Actin:

- Forward: 5'-GACTTAGTTGCGTTACACCCTT-3'

- Reverse: 5'-TTTTGACCTTGCCACTTCCA-3'
Flow Cytometry Analysis

The number of apoptotic cells in the different treatment groups was examined using flow cytometry analysis. The cells were stained with the Annexin V/fluorescein isothiocyanate (FITC) kit (BD Biosciences, Franklin Lakes, NJ, USA) according to the manufacturer's instructions.

\section{Infarct Volume Measurement}

Two-percent 2,3,5-triphenyltetrazolium chloride (TTC) staining was used to determine the infarct volume of the brain after reperfusion or sham operation. After being anesthetized, the brains were rapidly removed and froze in $-20^{\circ} \mathrm{C}$ for $30 \mathrm{~min}$ and then sliced into serial coronal sections ( $2-\mathrm{mm}$ thick). The sections were incubated in $2 \%$ TTC for $30 \mathrm{~min}$ at $37^{\circ} \mathrm{C}$. TTC-stained sections were photographed by using an Olympus microscope (Olympus, Tokyo, Japan). The infarcted area identified as unstained by TTC were measured by Image J software (National Institutes of Health, Bethesda, USA). The percentage of infarct size per slice was calculated as follows: (infarcted area/total brain area) $\times 100 \%$.

\section{Neurological Deficit Score Assessment}

Neurological deficit (Bederson scores) scores were determined at $24 \mathrm{~h}$ after MCAO according to the criteria of Longa 5 scores [18]. The neurological deficit was graded as follows: 0 score, nonneurological deficit; score 1, extending contralateral forelimb impairment; score 2, circling to the left; score 3, fall forward to the left; score 4 , no spontaneous walking and unconsciousness. The performer was blind to the treatments.

\section{Statistical Analysis}

Data were presented as the mean \pm SD. Experimental analysis was performed using GraphPad Prism 6.0 software (GraphPad Software, San Diego, CA, USA). Differences between groups were determined using Student's $t$ test or one-way ANOVA with a Bonferroni post hoc test. $p<0.05$ was considered to indicate a statistically significant difference.

\section{Results}

\section{Inhibition of AQP4 Could Alleviate Injury of}

Astrocyte Cells Caused by Ischemia Reperfusion

To identify the protection effect of AQP4 on the astrocyte cells after ischemia reperfusion, we established the CIRI and the OGD/RX model in vivo and in vitro to detect the changes in AQP4 expression, resulting that the expression of AQP4 significantly increased in a time-dependent manner after reperfusion at 24 and $48 \mathrm{~h}$ followed by CIRI or OGD/RX treatment (Fig. 1a). Then, we found that knockdown of AQP4 siRNA could significantly enhance the decrease in OGD/RX-induced cell viability (Fig. 1b; Table 1). The results of cytotoxicity LDH assay analysis showed that AQP4 interference could reduce the increase in OGD/RX-induced LDH release (Fig. 1c). Flow cytometry showed that the rate of apoptotic cells was re- 


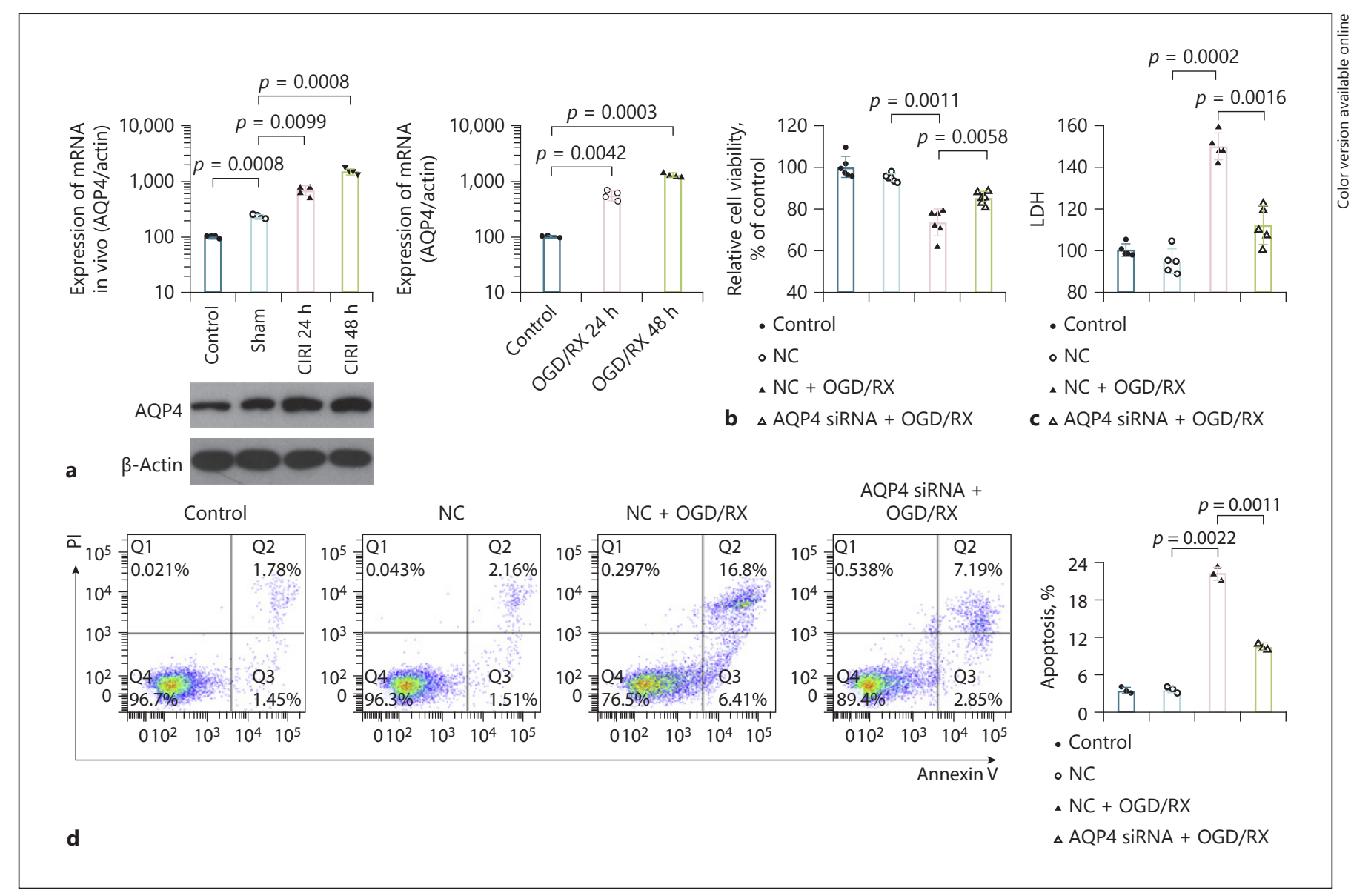

Fig. 1. AQP4 could promote the injury of astrocyte cells caused by ischemia reperfusion. a The expression of AQP4 mRNA was determined by RT-qPCR after CIRI for 24 and $48 \mathrm{~h}$, or OGD/RX for 24 and $48 \mathrm{~h}$. Western blot was performed to detect the expression of AQP4 after CIRI for 24 and 48 h, sham, or control. b A CCK-8 assay determining cell viability under OGD/RX condition following transfection with or without AQP4 siRNA. c LDH was deter-

duced after AQP4 siRNA treatment (Fig. 1d), indicating that AQP4 interference could protect astrocyte damage caused by OGD/RX.

\section{Absence of MALAT1 Could Protect OGD/RX-Induced Astrocyte Cell Injury}

It has been reported that lncRNA plays an important role in stroke [6]. Zhang et al. [19] found that upregulation of MALAT1 could protect brain injury by inhibiting AQP4. However, we found that the level of MALAT1 was upregulated after OGD-RX treatment for 24 and $48 \mathrm{~h}$, which was consistent with the findings of Zhang et al. [9] (Fig. 2a). Transfecting the cells with MALAT1 siRNA1, siRNA2, or siRNA3, we found that the expression of mined by LDH assay analysis in the NC + OGD/RX group and AQP4 siRNA + OGD/RX group. d The rate of apoptotic cells was detected by flow cytometry. AQP4, aquaporin-4; OGD/RX, oxygen-glucose deprivation/reoxygenation; CIRI, cerebral ischemia reperfusion injury; CCK-8, Cell Counting Kit-8; LDH, lactate dehydrogenase; TTC, 2,3,5-triphenyltetrazolium chloride.

Table 1. CCK-8 assay determined cell viability under OGD/RX condition following transfection with or without AQP4 siRNA

\begin{tabular}{lc}
\hline Groups & Mean \pm SD \\
\hline Control & $100 \pm 4.7589$ \\
NC & $94.9069 \pm 1.6626$ \\
NC + OGD/RX & $73.5119 \pm 5.9594^{\mathrm{a}}$ \\
AQP4 siRNA + OGD/RX & $85.5971 \pm 2.8636^{\mathrm{b}}$ \\
\hline
\end{tabular}

CCK-8, Cell Counting Kit-8; AQP4, aquaporin-4; OGD/RX, oxygen-glucose deprivation/reoxygenation. ${ }^{\mathrm{a}} p<0.01$ versus NC. ${ }^{\mathrm{b}} p<0.01$ versus $\mathrm{NC}+\mathrm{OGD} / \mathrm{RX}$. 


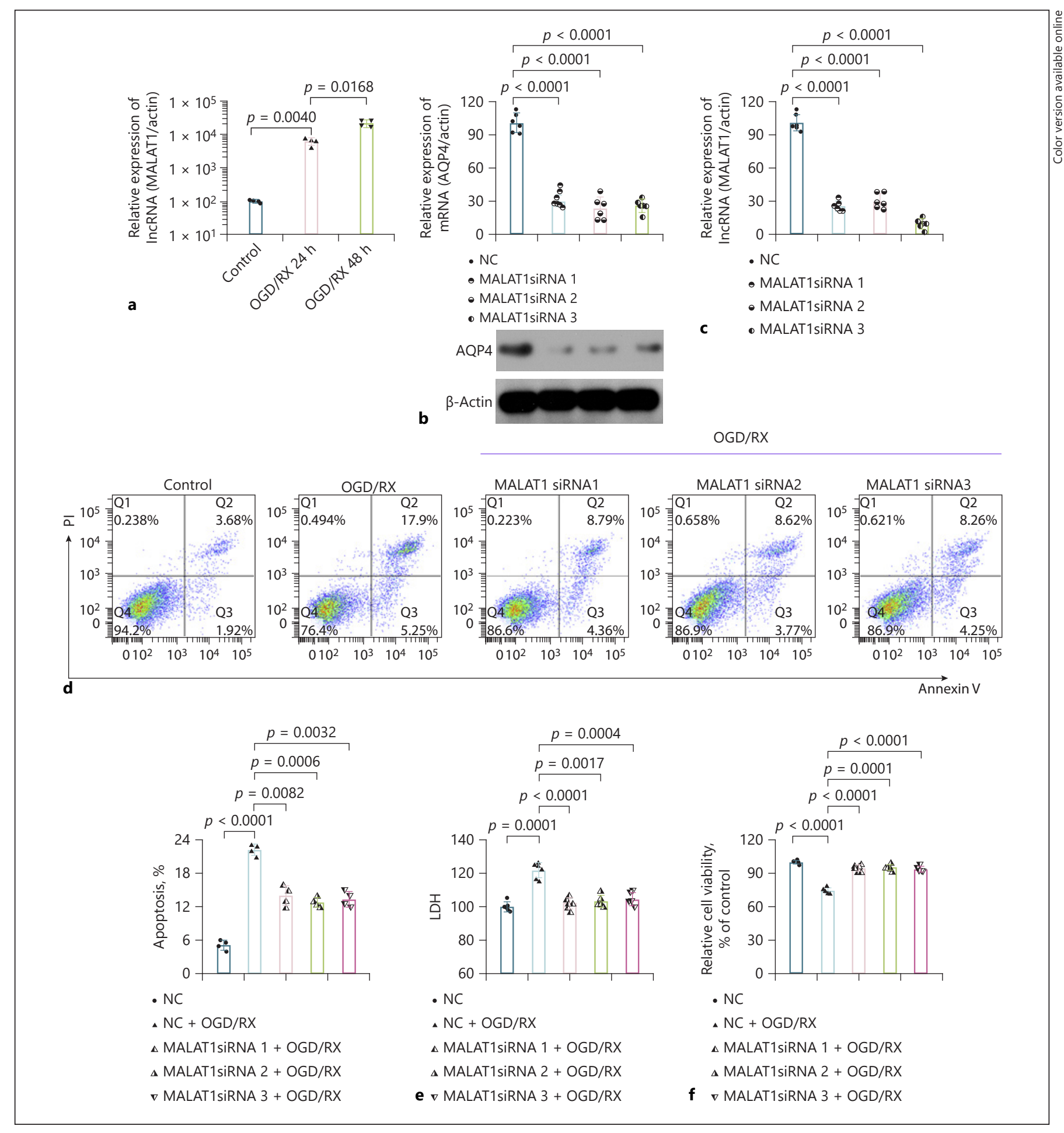

Fig. 2. Absence of MALAT1 could protect OGD/RX-induced astrocyte cell injury. a The level of MALAT1 was determined after OGD/RX for 24 and $48 \mathrm{~h}$. b The expression of AQP4 was detected by RT-qPCR after transfection with or without MALAT1 siRNA. c RT-qPCR determined the interference efficiency of MALAT1. d The rate of apoptotic cells was detected by flow cytometry. e $\mathrm{LDH}$ was determined by $\mathrm{LDH}$ assay analysis in the NC + OGD/
RX group and MALAT1 siRNA + OGD/RX group. f CCK-8 assay was used to examine cell viability under OGD/RX condition following transfection with or without MALAT1 siRNA. OGD/RX, oxygen-glucose deprivation/reoxygenation; AQP4, aquaporin-4; CIRI, cerebral ischemia reperfusion injury; CCK-8, Cell Counting Kit-8; LDH, lactate dehydrogenase; TTC, 2,3,5-triphenyltetrazolium chloride. 
Table 2. RT-qPCR determined the interference efficiency of MALAT1

\begin{tabular}{lc}
\hline Groups & Mean \pm SD \\
\hline NC & $100 \pm 6.7845$ \\
MALAT1 siRNA 1 & $25.0821 \pm 4.1905^{* * *}$ \\
MALAT1 siRNA 2 & $29.6534 \pm 6.2587^{* * *}$ \\
MALAT1 siRNA 3 & $9.1268 \pm 4.3294^{* * *}$ \\
\hline
\end{tabular}

$* * * p<0.001$ versus NC.

AQP4 protein and mRNA was significantly downregulated compared with NC using Western blot and RTqPCR analysis, respectively (Fig. 2b). The interference efficiency of MALAT1 siRNA was determined by RTqPCR (Fig. 2c; Table 2). Moreover, the rate of apoptotic cells and the LDH release was decreased following transfection with MALAT1 siRNA under OGD/RX condition (Fig. 2d, e; Table 3). CCK-8 assay analysis determined that cell viability significantly increased after MALAT1 siRNA treatment in OGD/RX (Fig. 2f; Table 4).

\section{The Protective Effect of MALAT1 on CIRI Injury in Rats}

To further demonstrate the protective effect of MALAT1 siRNA on brain injury in vivo, we first examined the level of MALAT1 under CIRI condition and found that compared with the sham group, the expression of MALATA was observed to downregulate in CIRI treatment for 24 and $48 \mathrm{~h}$ (Fig. 3a). After CIRI treatment, transfection with MALAT1 could significantly downregulate the Bedserson scores and the neurological behavior was also ameliorated by NC which was evaluated by the Bedserson score analysis (Fig. 3b). Western blot was performed to confirm the expression of AQP4 following CIRI treatment, which showed that compared with the CIRI group, the level of AQP4 was attenuated after treatment with MALAT1 siRNA under CIRI condition (Fig. 3c). The infarct volume evaluated by TCC analysis dramatically decreased after treatment with MALAT1 siRNA compared with the NC group under CIRI condition (Fig. 3d).

\section{Inhibition of MALAT1 May Play a Protective Role in}

Brain Stroke through Regulating AQP4

To verify whether MALAT1 plays an important role in brain injury via regulating $\mathrm{AQP} 4$, we transfected the cells with AQP4 plasmid or MALAT1 siRNA and observed the changes in cell viability, $\mathrm{LDH}$, and the number of apop-
Table 3. $\mathrm{LDH}$ was determined by $\mathrm{LDH}$ assay analysis in the $\mathrm{NC}+$ OGD/RX group and MALAT1 siRNA + OGD/RX group

\begin{tabular}{lc}
\hline Groups & Mean \pm SD \\
\hline NC & $100 \pm 2.9171$ \\
NC + OGD/RX & $121.8491 \pm 3.8064^{* * *}$ \\
MALAT1 siRNA 1 + OGD/RX & $102.0487 \pm 2.3808^{* * *}$ \\
MALAT1 siRNA 2 + OGD/RX & $103.1070 \pm 3.6223^{* * *}$ \\
MALAT1 siRNA3 + OGD/RX & $104.5681 \pm 3.8601^{* * *}$ \\
\hline
\end{tabular}

$\mathrm{LDH}$, lactate dehydrogenase; OGD/RX, oxygen-glucose deprivation/reoxygenation. ${ }^{* * *} p<0.001$ versus NC.

Table 4. CCK- 8 assay was used to examine cell viability under OGD/RX condition following transfection with or without MALAT1 siRNA

\begin{tabular}{lc}
\hline Groups & Mean \pm SD \\
\hline NC & $100 \pm 0.6900$ \\
NC + OGD/RX & $74.4844 \pm 2.635^{* * *}$ \\
MALAT1 siRNA 1 + OGD/RX & $94.6882 \pm 2.4581^{* * *}$ \\
MALAT1 siRNA 2 + OGD/RX & $95.4761 \pm 2.4073^{* * *}$ \\
MALAT1 siRNA3 + OGD/RX & $93.7332 \pm 1.2628^{* * *}$ \\
\hline
\end{tabular}

CCK-8, Cell Counting Kit-8; OGD/RX, oxygen-glucose deprivation/reoxygenation. ${ }^{* * *} p<0.001$ versus NC.

totic cells after OGD/RX. The results showed that AQP4 plasmid treatment could reduce cell viability and enhance $\mathrm{LDH}$ release and the rate of apoptotic cells, while with MALAT1 siRNA, there was no significant difference between these 2 groups; the protective role of MALAT1 siRNA vanished (Fig. $4 a-c)$. The transfection efficiency of MALAT1 siRNA and AQP4 plasmid was determined by RT-qPCR (Fig. 4d). AQP4 plasmid transfection efficiency was further verified by Western blot (Fig. 4e).

\section{Discussion}

MALAT1 is among the first lncRNAs identified as promoting metastasis and proliferation of different cancers through alternative splicing and gene expression [20, $21]$. It is a well-conserved and abundant lncRNA, existing in different species. A recent study had demonstrated that MALAT1 was expressed in vascular endothelial cells, skeletal muscle, gastric cancer, and colorectal cancer [2226]. Recently, the functions of MALAT1 in ischemic stroke were identified [10]. For example, downregulation 


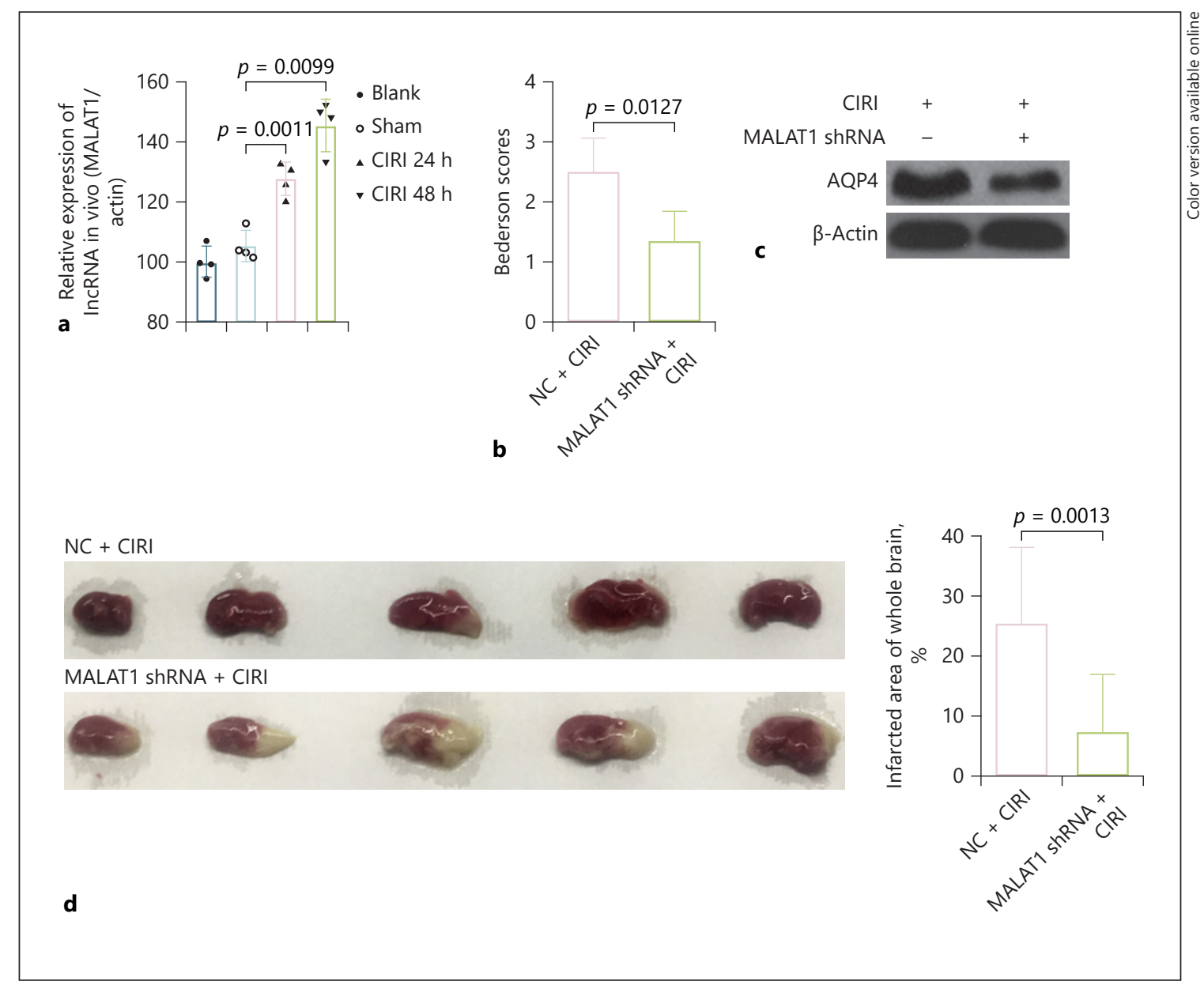

Fig. 3. Inhibition of MALAT1 level could protect brain injury and improve neuronal function in CIRI-treated mice. a MALAT1 expression under CIRI condition for 24 and $48 \mathrm{~h}$ was determined by RT-qPCR. $\mathbf{b}$ The role of interference of MALAT1 on the neuronal impairment was assessed by neurological deficit scores. c Western blot determined the expression of AQP4 was downregulated after transfection with MALAT1 siRNA under CIRI condition. d The effect of MALAT1 siRNA on the focal ischemia of brain sections and quantitative analysis of brain infarct volumes were shown by TTC staining in the CIRI model. CIRI, cerebral ischemia reperfusion injury; TTC, 2,3,5-triphenyltetrazolium chloride. of MALAT1 could attenuate neuronal cell death in cerebral ischemic stroke [27]. The present study found that MALAT1 was significantly upregulated after CIRI or OGD/RX for 24 and $48 \mathrm{~h}$. Inhibition of MALAT1 could alleviate OGD/RX-induced astrocyte cell injury through decreasing the ratio of apoptotic cells and the release of $\mathrm{LDH}$ and enhancing cell viability in vitro; furthermore, it could reduce the infarcted area of the whole brain and the neurological behavior.

AQP4 is considered as an appealing therapeutic target for brain disorders such as brain tumor, traumatic injury, hydrocephalus, and neuroinflammation [28-30]. It has been indicated that knockdown of AQP4 could improve patient outcomes and neurological function, reduce in- farction volume, increase neuronal survival, and reduce apoptosis and the inflammatory response following cerebral ischemia, in accordance with brain edema reduction [31]. AQP4 was also found to be overexpressed in the reactive astrocytes after cerebral injury [32], which is consistent with our findings. In addition, we found that AQP4 was upregulated after CIRI in vivo. Furthermore, our findings demonstrated that compared with the OGD/ $\mathrm{RX}$ group, absence of AQP4 could increase the decrease in OGD/RX-induced cell viability and decrease the release of LDH and the number of apoptotic cells, indicating that AQP4 interference may protect OGD/RX-induced astrocyte injury, which was consistent with the findings of Zheng et al. [16]. 

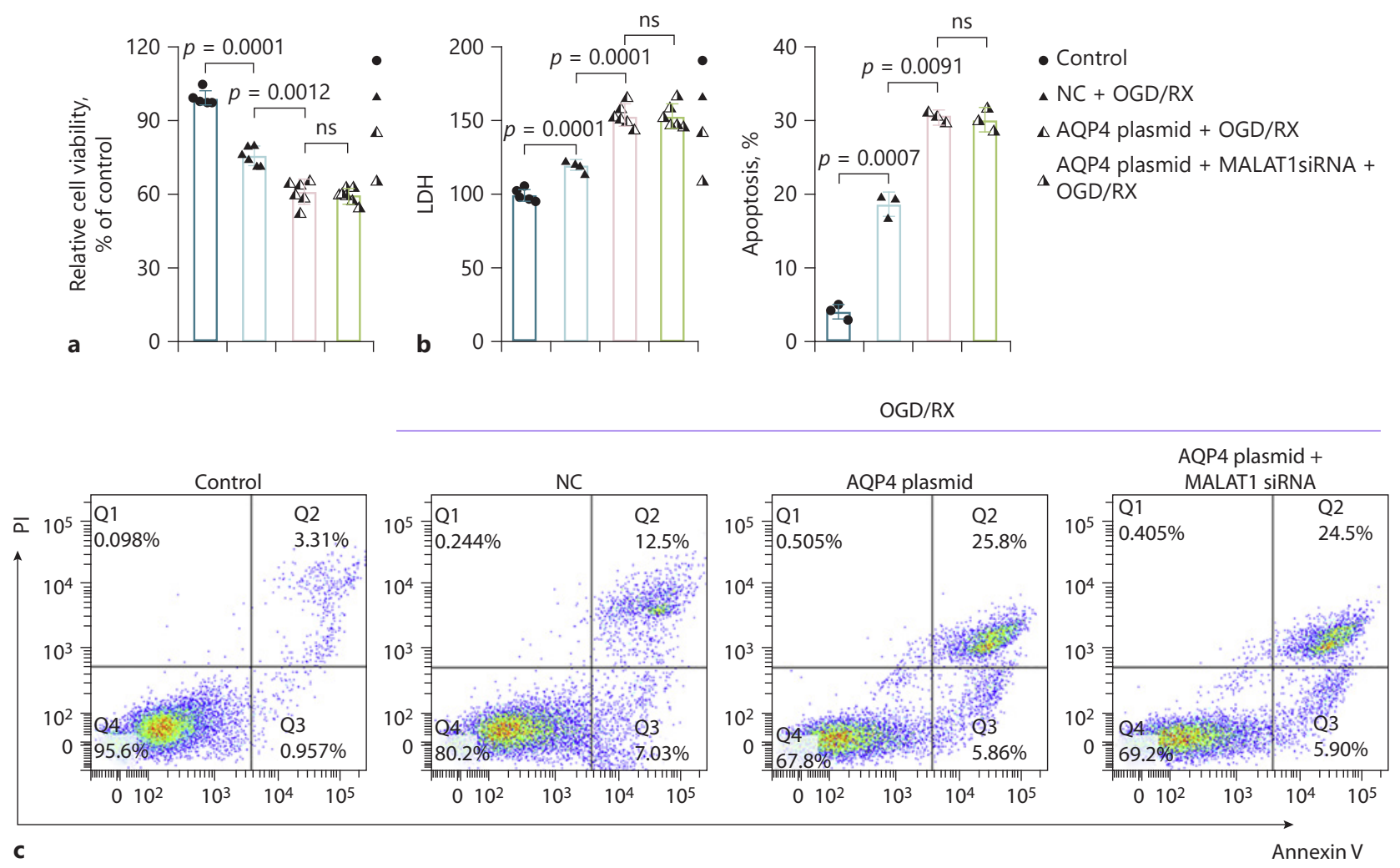

c

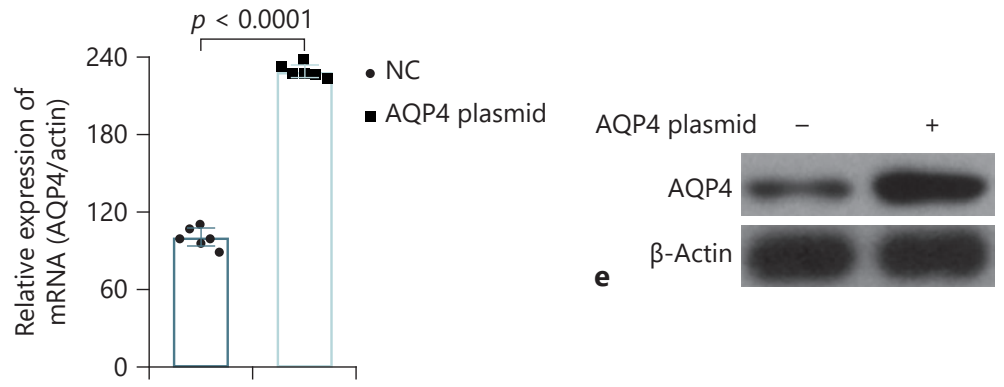

Fig. 4. Inhibition of MALAT1 may play a protective role in brain stroke through regulating AQP4. a A CCK-8 assay determined cell viability following transfection with $\mathrm{AQP} 4$ plasmid or AQP4 plasmid combined with MALAT1 siRNA under OGD/RX condition. b, c Flow cytometry analyses were used to determine the rate of apoptotic cells transfected with AQP4 plasmid or AQP4 plasmid

Recently, miRNA or LncRNA was identified to regulate AQP4 to protect cerebral ischemic reperfusion injury $[11,12,16,33]$. In the present study, we further explore that the protective effect of MALAT1 in cerebral ischemic reperfusion injury was related to AQP4 expression. Interestingly, inhibition of MALAT1 could downregulate AQP4 expression in vitro and in vivo. In addi- combined with MALAT1 siRNA under normal or OGD/RX conditions. d RT-qPCR analysis was used to determine the level of MALAT1 and AQP4. e The protein level of AQP4 transfected with AQP4 plasmid was determined by Western blot. AQP4, aquaporin-4; CCK-8, Cell Counting Kit-8; OGD/RX, oxygen-glucose deprivation/reoxygenation. 
In conclusion, our study revealed that inhibition of MALAT1 could reduce cerebral I/ $R$ injury in vitro and in vivo by suppressing the expression of AQP4. Therefore, MALAT1 may serve as a novel therapeutic target for the treatment of cerebral ischemic stroke.

\section{Statement of Ethics}

All animal experiments were approved by the First Affiliated Zhejiang Hospital, Zhejiang University of Medical Ethics Committee, and the Medical Faculty Ethics Committee of the First Affiliated Zhejiang Hospital, Zhejiang University, in accordance with the National Institutes of Health Guide for Care and Use of Laboratory Animals (NIH Publications, No. 8023, revised 1978).

\section{Conflict of Interest Statement}

The authors declare that they have no conflicts of interest.

\section{Funding Sources}

This study was funded by the Key Research \& Development Plan of Zhejiang Province (No. 2019C03034) and the Project of Health and Family Planning Commission of Zhejiang Province (2017KY327).

\section{Author Contributions}

Renya Zhan conceived the idea and wrote the manuscript; Jing Jin and Hongwei Wang performed the experiments; Xiaoxiao Zheng, Shangzhi Xie, and Li Zheng analyzed the data; and Jing Jin revised the manuscript. All authors have read and approved the final version of the manuscript.

\section{References}

1 Siniscalchi A, Gallelli L, Malferrari G, Pirritano D, Serra R, Santangelo E, et al. Cerebral stroke injury: the role of cytokines and brain inflammation. J Basic Clin Physiol Pharmacol. 2014;25(2):131-7.

2 Randolph SA. Ischemic stroke. Workplace Health Saf. 2016;64:444.

3 Gomis M, Dávalos A. Recanalization and reperfusion therapies of acute ischemic stroke: what have we learned, what are the major research questions, and where are we headed? Front Neurol. 2014;5:226.

4 Molina CA, Alvarez-Sabín J. Recanalization and reperfusion therapies for acute ischemic stroke. Cerebrovasc Dis. 2009;27(Suppl 1): $162-7$.

5 Ong SB, Katwadi K, Kwek XY, Ismail NI, Chinda K, Ong SG, et al. Non-coding RNAs as therapeutic targets for preventing myocardial ischemia-reperfusion injury. Expert Opin Ther Targets. 2018;22(3):247-61.

6 Bao MH, Szeto V, Yang BB, Zhu SZ., Sun HS, Feng ZP. Long non-coding RNAs in ischemic stroke. Cell Death Dis. 2018;9(3):281.

7 Chen H, Li X. LncRNA ROR is involved in cerebral hypoxia/reoxygenation-induced injury in pc12 cells via regulating miR-135a-5p/ ROCK1/2. Am J Transl Res. 2019;11(9):614558.

8 Wang J, Cao B, Zhao H, Gao Y, Luo Y, Chen $\mathrm{Y}$, et al. Long noncoding RNA h19 prevents neurogenesis in ischemic stroke through p53/ NOTCH1 pathway. Brain Research Bulletin. 2019;150:111-7.

9 Zhang J, Yuan L, Zhang X, Hamblin MH, Zhu $\mathrm{T}$, Meng $\mathrm{F}$, et al. Altered long non-coding RNA transcriptomic profiles in brain microvascular endothelium after cerebral ischemia. Exp Neurol. 2016;277:162-70.
10 Zhang X, Tang X, Liu K, Hamblin MH, Yin $\mathrm{KJ}$. Long noncoding RNA MALAT1 regulates cerebrovascular pathologies in ischemic stroke. J Neurosci. 2017;37(7):1797-806.

11 Zheng L, Cheng W, Wang X, Yang Z, Zhou X, Pan C. Overexpression of microRNA-145 ameliorates astrocyte injury by targeting aquaporin 4 in cerebral ischemic stroke. Biomed Res Int. 2017;2017:9530951.

12 Zheng Y, Wang L, Chen M, Pei A, Xie L, Zhu $\mathrm{S}$. Upregulation of miR-130b protects against cerebral ischemic injury by targeting water channel protein aquaporin 4 (AQP4). Am J Transl Res. 2017;9(7):3452-61.

13 Manley GT, Fujimura M, Ma T, Noshita N, Filiz F, Bollen AW, et al. Aquaporin-4 deletion in mice reduces brain edema after acute water intoxication and ischemic stroke. Nat Med. 2000;6(2):159-63.

14 Iwata-Ichikawa E, Kondo Y, Miyazaki I, Asanuma M, Ogawa N. Glial cells protect neurons against oxidative stress via transcriptional up-regulation of the glutathione synthesis. J Neurochem. 1999;72(6):2334-44.

15 Lin SP, Ye S, Long Y, Fan Y, Mao HF, Chen MT, et al. Circular RNA expression alterations are involved in OGD/R-induced neuron injury. Biochem Biophys Res Commun. 2016; 471(1):52-6.

16 Zheng Y, Pan C, Chen M, Pei A, Xie L, Zhu S. miR-29a ameliorates ischemic injury of astrocytes in vitro by targeting the water channel protein aquaporin 4. Oncol Rep. 2019;41(3): 1707-17.

17 Livak KJ, Schmittgen TD. Analysis of relative gene expression data using real-time quantitative PCR and the 2(-delta delta C(T)) method. Methods. 2001;25(4):402-8.
18 Longa EZ, Weinstein PR, Carlson S, Cummins R. Reversible middle cerebral artery occlusion without craniectomy in rats. Stroke. 1989;20(1):84-91.

19 Zhang Y, Wang J, Zhang Y, Wei J, Wu R, Cai $\mathrm{H}$. Overexpression of long noncoding RNA MALAT1 ameliorates traumatic brain injury induced brain edema by inhibiting AQP4 and the NF- $\kappa B / I L-6$ pathway. J Cell Biochem. 2019;120(10):17584-92.

20 Alishahi M, Ghaedrahmati F, Kolagar TA, Winlow W, Nikkar N, Farzaneh M, et al. Long non-coding RNAs and cell death following ischemic stroke. Metab Brain Dis. 2019;34(5): 1243-51.

21 Ji P, Diederichs S, Wang W, Böing S, Metzger $\mathrm{R}$, Schneider PM, et al. MALAT-1, a novel noncoding RNA, and thymosin beta4 predict metastasis and survival in early-stage nonsmall cell lung cancer. Oncogene. 2003; 22(39):8031-41.

22 Michalik KM, You X, Manavski Y, Doddaballapur A, Zörnig M, Braun T, et al. Long noncoding RNA MALAT1 regulates endothelial cell function and vessel growth. Circ Res. 2014;114(9):1389-97.

23 Tang Y, Jin X, Xiang Y, Chen Y, Shen CX, Zhang YC, et al. The IncRNA MALAT1 protects the endothelium against ox-LDL-induced dysfunction via upregulating the expression of the miR-22-3p target genes CXCR2 and AKT. FEBS Lett. 2015;589(20 Pt B):3189-96.

24 Yong $\mathrm{H}, \mathrm{Wu}$ G, Chen J, Liu X, Bai Y, Tang N, et al. IncRNA MALAT1 accelerates skeletal muscle cell apoptosis and inflammatory response in sepsis by decreasing BRCA1 expression by recruiting EZH2. Mol Ther Nucleic Acids. 2020;19:97-108. 
25 YiRen H, YingCong Y, Sunwu Y, Keqin L, Xiaochun T, Senrui C, et al. Long noncoding RNA MALAT1 regulates autophagy associated chemoresistance via miR-23b-3p sequestration in gastric cancer. Mol Cancer. 2017;16(1):174.

26 Li P, Zhang X, Wang H, Wang L, Liu T, Du L, et al. MALAT1 is associated with poor response to oxaliplatin-based chemotherapy in colorectal cancer patients and promotes chemoresistance through EZH2. Mol Cancer Ther. 2017;16(4):739-51.

27 Guo D, Ma J, Yan L, Li T, Li Z, Han X, et al. Down-regulation of IncRNA MALAT1 attenuates neuronal cell death through suppressing beclin1-dependent autophagy by regulating
miR-30a in cerebral ischemic stroke. Cell Physiol Biochem. 2017;43(1):182-94.

28 Zhao J, Moore AN, Clifton GL, Dash PK. Sulforaphane enhances aquaporin-4 expression and decreases cerebral edema following traumatic brain injury. J Neurosci Res. 2005; 82(4):499-506.

29 Li L, Zhang H, Varrin-Doyer M, Zamvil SS, Verkman AS. Proinflammatory role of aquaporin-4 in autoimmune neuroinflammation. FASEB J. 2011;25(5):1556-66.

30 Frydenlund DS, Bhardwaj A, Otsuka T, Mylonakou MN, Yasumura T, Davidson KG, et al. Temporary loss of perivascular aquaporin-4 in neocortex after transient middle cere- bral artery occlusion in mice. Proc Natl Acad Sci U S A. 2006;103(36):13532-6.

31 Tang G, Yang GY. Aquaporin-4: a potential therapeutic target for cerebral edema. Int J Mol Sci. 2016;17.

32 Rao KV, Reddy PV, Curtis KM, Norenberg $\mathrm{MD}$. Aquaporin-4 expression in cultured astrocytes after fluid percussion injury. J Neurotrauma. 2011;28(3):371-81.

33 Shan W, Chen W, Zhao X, Pei A, Chen M, Yu $\mathrm{Y}$, et al. Long noncoding RNA TUG1 contributes to cerebral ischaemia/reperfusion injury by sponging miR-145 to up-regulate AQP4 expression. J Cell Mol Med. 2020 Jan;24(1): 250-59. 